\title{
Unmoderated Posters: Sexual Function and Dysfunction
}

\section{UP-52}

Current Management of Peyronie's Disease among Urological Consultants in the United Kingdom National Health Service Al-Qassim, Zubair'; Mohammed, Aza'; Payne, David'; England, Roland'; Summerton, Duncan ${ }^{2}$

${ }^{1}$ Kettering General Hospital, Kettering, Northamptonshire, United Kingdom; ${ }^{2}$ Leicester General Hospital, Leicester, Leicestershire, United Kingdom Objectives: To identify the current trends in the management of Peyronie's Disease (PD) in the UK National Health Service (NHS) and to identify any differences in the practice between University Hospitals (UH) and District General Hospitals (DGH).

Methods: A questionnaire was emailed to consultant urologists currently practicing in the UK. The data was analyzed using descriptive statistics and Fisher's exact test.

Results: 181 consultants responded with $46.4 \%$ working at DGH and $53.6 \%$ at UH. Half of the respondents had 10 years or more experience as consultants with a third of consultants in both hospital types had seen 10 or more new cases of PD in the last 12 months. The majority used simple analgesia or vitamin E for acute phase PD. More patients with stable disease were treated in DGH compared to UH. Methods to assess the degree of curvature included digital photography, patient's own account and artificial erection. PD with higher degrees of curvatures ( $>60$ degrees) for both dorsal and ventral deformities tend to be operated more in $U \mathrm{H}$. Penile straightening procedures were the predominant operations performed with the tendency for incision of the corpora rather than simple plication in Nesbit's operation. For plague incision/excision and grafting, synthetic materials were used more in DGH compared to UH (38.5\% vs. $20 \%)$, while autologus materials are more used in UH $(52 \%$ and $28 \%$ vs. $38.5 \%$ and $23.1 \%$ ). Inflatable implants were the main type of prostheses used and were performed more in UH compared to malleable implants that were performed more in DGHs. The majority of the consultants in both hospital types would perform the complex procedures (plague incision/excision and graft and implants) independently with minimal participation from urology specialist registrars.

Conclusions: The management of PD when compared between DGH and $\mathrm{UH}$ on the whole appears to show similar practices. The main differences seem to centre around surgical operations for more complex deformities where the degrees of curvature are greater. As the questionnaire focused on management issues rather than outcomes, we advocate a prospectively collected British Association of Urological Surgeons database on PD patients to take this interesting disease entity forward.

\section{UP-53}

Variance of Urosurgeon Reimbursement for Inflatable Penile Prosthesis Surgery: Analyses of Provincial Reimbursement Schedules and Comparison to Artificial Urinary Sphincter Implantation

Bella, Anthony 1.; De Jesus, Anthony

University of Ottawa, Ottawa, ON, Canada

Introduction and Objectives: The role of the inflatable penile prosthesis (IPP) as definitive management of refractory to first and second line erectile dysfunction is well established, as treatment confers significant patientreported quality-of-life. Reimbursement rates for IPP surgery are established on a province-by-province basis. We report on the wide variance for urosurgeon reimbursement across Canada.

Methods: In-depth analyses utilizing electronically accessed provincial reimbursement fee schedules was performed for IPP surgery across Canada. Comparisons were performed interprovincially, as well as in relation to artificial urinary sphincter (AUS) implantation, a technically similar surgery requiring placement of a fluid reservoir, dissection to the urethra/corpora, and surgeon technical skills shared between IPP and AUS placement.

Results: Surprisingly, there was significant variability for provincial reimbursement, which did not follow recent CIHR per capita health spending patterns on physician reimbursement. Most provinces reimbursed between $\$ 600$ and $\$ 760$ for IPP placement, with AUS placement generally reimbursed \$100-200 more (up to \$943.09). Ontario reimbursed the least for the IPP (\$362), while AUS reimbursement was $\$ 776.70$, for the widest differential between the two procedures for provinces where coverage is in place for both.

Conclusions: The role of the IPP, especially as the final step in prostate cancer survivorship when addressing oncology treatment-related side-effects, is clearly defined in the appropriate patient. In addition to traditional patient barriers to care, the economics of urosurgeon reimbursement may limit access to IPP procedures, especially as the complexity of surgery increases. Critically evaluating reimbursement with adjustment to fee schedules as necessary may be seen as equitable within the urologic community. 